\title{
The Design and Development of a High-Throughput Magneto-Mechanostimulation Device for Cartilage Tissue Engineering
}

\author{
Mariea A. Brady, PhD, Reva Vaze, MSc, Harsh D. Amin, PhD, \\ Darryl R. Overby, $\mathrm{PhD}^{1}$, and C. Ross Ethier, $\mathrm{PhD}^{1,2}$
}

To recapitulate the in vivo environment and create neo-organoids that replace lost or damaged tissue requires the engineering of devices, which provide appropriate biophysical cues. To date, bioreactors for cartilage tissue engineering have focused primarily on biomechanical stimulation. There is a significant need for improved devices for articular cartilage tissue engineering capable of simultaneously applying multiple biophysical (electrokinetic and mechanical) stimuli. We have developed a novel high-throughput magnetomechanostimulation bioreactor, capable of applying static and time-varying magnetic fields, as well as multiple and independently adjustable mechanical loading regimens. The device consists of an array of 18 individual stations, each of which uses contactless magnetic actuation and has an integrated Hall Effect sensing system, enabling the real-time measurements of applied field, force, and construct thickness, and hence, the indirect measurement of construct mechanical properties. Validation tests showed precise measurements of thickness, within $14 \mu \mathrm{m}$ of gold standard calliper measurements; further, applied force was measured to be within $0.04 \mathrm{~N}$ of desired force over a half hour dynamic loading, which was repeatable over a 3-week test period. Finally, construct material properties measured using the bioreactor were not significantly different $(p=0.97)$ from those measured using a standard materials testing machine. We present a new method for articular cartilage-specific bioreactor design, integrating combinatorial magnetomechanostimulation, which is very attractive from functional and cost viewpoints.

\section{Introduction}

O SteoArthritis (OA), a Degenerative disease characterized by the erosion of articular cartilage, is a large and growing public health problem. OA currently affects more than 200 million people worldwide, 27 million of whom reside in the USA. ${ }^{1-4}$ Total joint replacement is the gold standard surgical treatment for the disease, with an associated economic cost in the USA of $\sim \$ 42.3$ billion per annum. ${ }^{4}$ A desire to provide more effective therapies has motivated efforts to develop tissue-engineering and regenerative medicine technologies to treat degenerative and traumatic joint surface defects. ${ }^{1}$ Functional tissue engineering is a promising approach that employs cells propagated in vitro to cellularize scaffolds; coupled with appropriate physical conditioning, this approach can establish tissue functionality before implantation. ${ }^{5}$

To identify suitable physical conditioning regimens for tissue-engineered constructs, it is necessary to understand the in vivo biochemical and mechanical environment of ar- ticular cartilage. ${ }^{1}$ Dynamic and transient mechanical loading of cartilage induces streaming and diffusion potentials, due to the flow of ions through the charged extracellular matrix. ${ }^{6}$ Streaming potentials can be modulated by cyclic loading conditions and by alterations in the proteoglycan charge or content, thus constituting a signaling mechanism that reflects both the mechanical environment and electrokinetic state of the extracellular matrix. ${ }^{7}$ Similarly, applying an electric potential or current to cartilage can produce stress and deformation in the tissue. ${ }^{6,8}$ Thus, chondrocytes clearly reside in, and respond to, an environment where they are exposed to electromechanical signals, including stresses, strains, and electric fields generated inside the extracellular matrix when the tissue is deformed. ${ }^{9}$

It has been noted that articular cartilage research to date has resulted in many publications on the effects of mechanical loading, with relatively few studies focused on the effect of fluid and ion flows, and electrical potentials within the extracellular matrix (ECM), where chondrocytes reside. ${ }^{9}$ This has been reflected in the design of bioreactors for cartilage

\footnotetext{
${ }^{1}$ Department of Bioengineering, Imperial College London, South Kensington, London, United Kingdom.

${ }^{2}$ Wallace H. Coulter Department of Biomedical Engineering, Georgia Institute of Technology, Atlanta, Georgia.
} 
tissue engineering. Since 1939, when Glucksmann built the first mechanostimulation device, many devices have been developed to apply mechanical stimuli to cartilage explants and engineered constructs. Lujan et al. ${ }^{10}$ reported over 205 cartilage engineering articles, which applied mechanical stimulation, $29 \%$ of which had the capacity to quantify material properties. Interestingly, most of these devices are only capable of applying a single type of mechanical stimulus, including compressive loading, ${ }^{11-17}$ bending or tensile stress, ${ }^{18-21}$ shear stress, ${ }^{22-24}$ and hydrostatic pressure. ${ }^{25-27}$ Of these modes of mechanical stimulation, compressive loading is considered to be the most important form of loading to act on cartilage in vivo. ${ }^{1}$ Further, many of these devices are limited to applying mechanical stimuli to a single specimen at any given time. A major limitation of a number of mechanostimulation devices is chamber sterility, as the load platen is often mechanically connected to an external actuator, thus requiring open culture conditions. The use of contactless actuation would be advantageous, as it would enable completely closed, sterile culture conditions to be maintained, both during loading setup and long-term culture.

Nevertheless, significant progress has been made using these devices to advance our understanding of the magnitude and distribution of biophysical forces and flows in the neighborhood of the chondrocytes within cartilage. However, to the best of the authors' knowledge, no device exists that uses a combinatorial approach to try to better recapitulate the in vivo environment of the chondrocyte in a flexible and modular manner.

A new paradigm for articular cartilage-specific bioreactors would be to combine two biophysical stimuli: magnetic fields (which elicit electrokinetic effects) and mechanical compression, into a single device. Furthermore, the large number of factors in the cellular milieu that can impact biological function necessitates the development of highthroughput approaches to study cell behavior in vitro. ${ }^{28}$
Therefore, our goal was to improve the bioreactor design by developing the first high-throughput device for magnetomechanobiology, ${ }^{29}$ capable of stimulating many cellembedded 3D matrices or tissues in a parallel and scalable arrangement, such that each matrix can be stimulated with a unique magneto or mechanical loading regimen (amplitude, frequency, or mode) and assayed independently.

\section{Materials and Methods}

\section{Design concept}

To enable systematic and dosimetric studies of the response of articular chondrocytes to multiple biophysical stimulation regimes, our high-throughput magnetomechanostimulation bioreactor (Fig. 1) was designed to be able to control the magnitude, frequency, duty cycle, duration, and mode of biophysical stimuli. Two modes of biophysical stimulation are possible: magneto-stimulation (static and time-varying magnetic fields; Fig. 1A) and mechanostimulation (static and dynamic loading; Fig. 1B). The bioreactor was built on an array concept, in which, multiple tissue-engineered constructs could be cultured within individual chambers. These chambers are placed on top of individual platforms, which have a magnetic actuator beneath them. The magnetic actuator consists of a permanent magnet, denoted the actuating magnet, affixed to the shaft of a linear actuator; a second permanent magnet, denoted the chamber magnet, is positioned inside the bioreactor chamber as the load applicator. The distance between the actuating magnet and chamber magnet, and hence the mechanical force on each sample, is independently controllable. The actuating magnet is cycled by a linear actuator (stepper motor) so as to produce a time-varying force on the chamber magnet, which will in turn deliver this force to the cultured tissue or matrix. A design in which the oscillating magnetic field was created using solenoids with variable current was
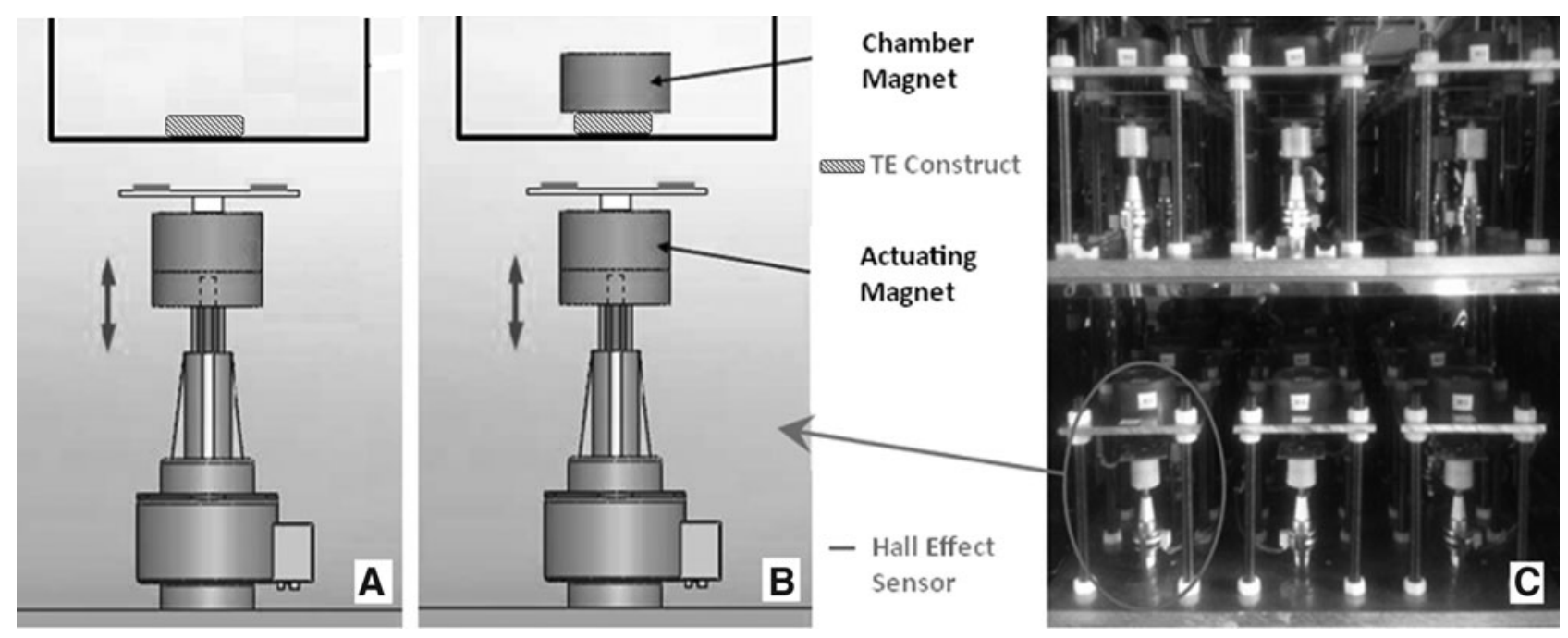

FIG. 1. The developed high-throughput magneto-mechanostimulation bioreactor can apply two modes of biophysical stimulation: (A) magneto-stimulation (static and time-varying magnetic fields); and (B) mechano-stimulation (static and dynamic loading). The device consists of actuating (contactless magnetic coupling) and sensing (Hall Effect sensor) components. Dynamic mechanical loading is enabled through the cyclic coupling of a permanent magnet pair via the extension and retraction of each linear actuator (as depicted by the green arrow in the schematics). Similarly, application of timevarying magnetic fields is achieved through cyclic extension and retraction of the linear actuator. The device is capable of stimulating 18 articular cartilage constructs independently and simultaneously (C). 
considered; however, this approach suffered from the requirement that the solenoid current be very large, which led to overheating. We therefore adopted the current permanent magnet approach. A critical design criterion was that the permanent magnets be adequately spaced or shielded so as not to influence the force on neighboring wells (Fig. 1C).

\section{Bioreactor construction}

The bioreactor consists of 18 individual Ultem ${ }^{\circledR}$ polyetherimide (PEI) 1000 (Elder Engineering (Herts) Ltd.) chambers. Ultem PEI is an amber transparent polymer commonly used in medical devices and was selected as it retains its material properties after 2000 autoclave cycles, ${ }^{30}$ enabling repeated sterilization as required in a tissue culture environment. Each chamber has dimensions of outer $\varnothing 53 \times 26 \mathrm{~mm}$, with a central cavity of diameter $37.5 \mathrm{~mm}$ and depth $24 \mathrm{~mm}$ (from the top surface). A wider 46-mm diameter hole is bored $6.5 \mathrm{~mm}$ deep, enabling a polycarbonate transwell ${ }^{\circledR}$ insert (VWR International Ltd.) to be placed in the chamber. Each chamber contains two openings, outer diameter $2 \mathrm{~mm}$, for fluid ingress and egress, enabling both static and perfusion culture conditions. A standard tissue culture 50-mm Petri dish lid is used to cover the chambers.

Each chamber is positioned centrally on a square aluminium top plate, of dimensions $110 \times 110 \times 6 \mathrm{~mm}$. This plate has a 2-mm groove for a PEI chamber to slot into. The top plate is then mounted on an aluminium base plate, via 10$\mathrm{mm}$-diameter brass studding, $160 \mathrm{~mm}$ in length. There are two aluminium base plates, denoted top shelf and bottom shelf, of dimension $420 \times 420 \times 12 \mathrm{~mm}$. Each base plate contains a three by three array of holes $(\varnothing 17 \mathrm{~mm})$, which house nine individually controllable Haydon-Kerk G4 stepper motor linear actuators (Reliance Precision Mechantronics LLP). The linear travel per full step is $102 \mu \mathrm{m}$ and a highresolution step movement is attained using $\mu$-step control, where $64 \mu$-steps $=1$ full step. Therefore, the linear distance moved per $\mu$-step is given by $\frac{\text { steps }}{64 \mu s t e p s} \times 0.102 \mathrm{~mm}$.

The upper chamber magnet is the load applicator, which is controlled in its vertical position in the chamber by means of the externally located, contactless actuating magnet attached to the stepper motor. An absolutely vertical compression can be ensured by centrally aligning the chamber magnet using a centering device. This device is made from the Ultem PEI rod, outer diameter $46 \mathrm{~mm}$ and length $18 \mathrm{~mm}$. A central cavity, which serves to contain and align the chamber magnet, is bored to a 13-mm depth, with outer diameter $24 \mathrm{~mm}$ and inner diameter $20 \mathrm{~mm}$. Four air holes in the upper segment of the centering device enable gas exchange inside the chambers.

The bioreactor, and all components used therein, are biologically and chemically inert and can be autoclaved. Furthermore, all bioreactor components, except the actuating magnets, are made of nonmagnetic materials. To enable optimal temperature, air humidity and composition, the bioreactor assembly is situated in a temperature-controlled and gas-regulated incubator (Sanyo $\mathrm{CO}_{2}$ cell culture incubator, MCO-18M; Sanyo).

\section{Biocompatibility of PEI chambers}

The biocompatibility of the PEI chambers was assessed against standard tissue culture treated six-well plates (WP). Bovine chondrocytes (BCs) were isolated as described by
McCullen et al. ${ }^{31}$; briefly, bovine cartilage was harvested from the lower leg joint of young calves. Chondrocytes were isolated by digesting cartilage in the Dulbecco's modified Eagle's medium (DMEM) + glutamax ( $4.5 \mathrm{~g} / \mathrm{L}$ glucose) with $0.2 \% \mathrm{w} / \mathrm{v}$ pronase, $10 \mathrm{mM}$ HEPES, and $50 \mathrm{mg} / \mathrm{mL}$ gentamicin (all reagents from Invitrogen) for $1 \mathrm{~h}$ at $37^{\circ} \mathrm{C}$ with agitation. This digest was removed and replaced with the DMEM+glutamax (4.5 g/L glucose) supplemented with $10 \mathrm{mM}$ HEPES, $50 \mathrm{mg} / \mathrm{mL}$ gentamicin, $5 \% \mathrm{v} / \mathrm{v}$ fetal bovine serum (FBS) (Invitrogen), and $0.04 \% \mathrm{w} / \mathrm{v}$ collagenase type I (Sigma) overnight at $37^{\circ} \mathrm{C}$ with agitation. After digestion, isolated chondrocytes were filtered through a $70-\mu \mathrm{m}$ pore size filter, centrifuged at $250 \mathrm{~g}$ for $3 \mathrm{~min}$, and plated in the DMEM ( $4.5 \mathrm{~g} / \mathrm{L}$ glucose) with $10 \% \mathrm{v} / \mathrm{v}$ FBS, $50 \mathrm{mg} / \mathrm{mL}$ ascorbic acid (Sigma), and $50 \mathrm{mg} / \mathrm{mL}$ gentamicin.

Free-swelling BC-seeded ( 2 million/mL) agarose hydrogels $(2.0 \mathrm{wt} \%$; Sigma) were then cultured for 2 weeks in chondrogenic media (DMEM-high glucose, $40 \mu \mathrm{g} / \mathrm{mL}$ L-proline [Sigma], $5 \mu \mathrm{g} / \mathrm{mL}$ ascorbate-2-phosphate [Sigma], ITS [BD Biosciences], $1 \%$ p/s, 10 ng/mL TGF- $\beta 3$ [Peprotech]) in both six WP and bioreactor chambers. The mechanical properties (compressive modulus) of the gels were measured using an Instron tester (see Material Property Evaluation section on next page) and the sulfated glycosaminoglycan (sGAG) content, a key extracellular matrix component of cartilage, was quantified (Blyscan GAG assay kit; Biocolor).

\section{Rare earth magnets and the force between magnet pairs}

A total of 36 (or 18 pairs), $0.4 \mathrm{~T}$ neodymium iron boron (NdFeB) magnets (Grade N42; Ø20mm, $10 \mathrm{~mm}$ height) are utilized in the bioreactor. $\mathrm{NdFeB}$ magnets were selected due to their innate performance characteristics: they have high coercive force, excellent demagnetization resistance capability, are cost effective, and are highly repeatable. Due to the fact that these magnets were to be placed in a cell culture, a bioinert gold coating (electrochemical plating) was applied; electrochemical plating was employed by the manufacturer (E-Magnets). Further, a PEI magnet holder was designed to contain the magnet, which inserts into the centering device as a secondary measure of protection in the case of any microcracks in the coating.

Magnetic field strength was measured using a Gaussmeter (GM08; Hirst Magnetic Instruments Ltd.). The probe was positioned on the central axis of the magnet (according to convention), flush against the magnet surface. The magnet was then moved away from the probe incrementally in steps of $102 \mu \mathrm{m}$ over a total distance of $50 \mathrm{~mm}$. Gaussmeter readings were taken at each position. The data obtained were used as a look up table for magneto-stimulation regimes in the bioreactor controller. For magneto-stimulation regimens, the user can input the required magnitude of the magnetic field (whether static or time varying) and the controller will adjust the distance the linear actuator is required to move to reach the user-defined field strength.

The force between each magnet pair is proportional to the distance between the magnets and was empirically determined using a standard materials testing machine (Model 5866 Instron), equipped with a 500-N load cell. Tensile grips were used to clasp each magnet of a pair, and then compression extension mode was used at a crosshead speed of 
$100 \mu \mathrm{m} / \mathrm{s}$. The data collected were used as a force-distance look-up table in the bioreactor controller for mechanostimulation regimens.

\section{Noncontact magnetic actuation and motor control}

As previously described, in our high-throughput bioreactor, the chamber magnet acts as the load applicator (or platen) and is situated atop the cell-seeded construct being cultured in each individual chamber. The contactless controlling of the top chamber magnet is carried out by an external actuating magnet housed in a white nylon (RS Components Ltd.) cylindrical holder, which is mounted on the shaft of the linear actuator stepper motor. Cyclic compressive loading is achieved by extension and retraction of the linear actuator shaft to the desired position (force) and at the required speed (frequency). As the shaft is extended, the actuating magnet attracts the chamber magnet, resulting in a compressive load being applied to the tissue construct located beneath the magnet. As the actuating magnet is retracted, the upper magnet is released.

The 18 stepper motor linear actuators are controlled using $3 \times$ Trinamic motion controller TMCM-612/SG (Micromech Ltd.) boards. Each TMCM-612/SG is a six axis two-phase stepper motor controller and driver module. The module provides a high micro ( $\mu$ )-step resolution to do very exact positioning and measurement tasks. Further, the module has a high-performance data acquisition capacity with an integrated, 8 channel, 16 bit ADC converter, which can be programmed to do a step-synchronous input voltage scan and store values at a high data rate. The measurement results are transferred to the PC using the high-speed USB interface. Further, a number of analog output channels and digital I/ Os can be used to control further instrumentation.

\section{Sensing system}

Precision Hall Effect (HE) position sensors were integrated into the bioreactor as a robust sensing system. HE sensors are applied as contact-less sensors for linear position, angular position, velocity, rotation, and current. ${ }^{32}$ The bioreactor utilizes the concept of a bias magnet, whereby the HE sensors (Allegro ${ }^{\circledR}$ [A1360, A1361, and A1362] Programmable linear HE sensor ICs) are mounted on the actuating magnet (at a distance of $11 \mathrm{~mm}$ ), and are fixed relative to the stepper motor. This enables the detection of the change in a magnetic field due to the motion of the upper chamber magnet as it moves toward the actuating (bias) magnet. The HE sensors were programmed according to the manufacturer's instructions and calibrated to produce an output voltage proportional to the distance between the magnet pair, denoted $\mathrm{D}_{\text {mag. }}$. The device sensitivity is adjustable within three guaranteed ranges: $0.7-1.4 \mathrm{mV} / \mathrm{G}$ (A1360), $1.4-4.5 \mathrm{mV} / \mathrm{G}$ (A1361), and 4.5-16 mV/G (A1362).

Before the integrated sensing system was calibrated, the geometry of the system and associated algorithms for calculating force and thickness were established. From the geometry of the system (Fig. 2) and known stepper motor position, a simple algorithm was derived that enabled indirect real-time measurements of both the applied force (a function of distance between a magnet pair; $\mathrm{D}_{\text {mag }}$ ) and construct thickness $\left(\mathrm{T}_{\mathrm{c}}\right)$. With reference to Figure 2, the following holds can be seen:

$$
\begin{gathered}
115.5+6+T_{c}=D_{\text {mag }}+\left(\frac{\text { steps }}{64 \mu \text { steps }} \times 0.102\right)+15+63.23 \mathrm{~mm} \\
\therefore T_{c}=D_{\text {mag }}+\left(\frac{\text { steps }}{64 \mu \text { steps }} \times 0.102\right)-43.27 \mathrm{~mm}
\end{gathered}
$$

The integrated HE position sensors, which produce an output voltage proportional to field strength/distance, provide the real-time measure of $\mathrm{D}_{\text {mag }}$, and subsequently, a realtime measure of magnetic field strength, force, and thickness from established look-up tables and derived algorithms. The HE sensors were easily programmed according to the manufacturer's instructions and calibrated to the system requirements, as follows. The stepper motors were incrementally moved by an absolute distance of $408 \mu \mathrm{m}$ ( $256 \mu$-steps), an average of 50 samples was taken at each position. Figure 3 shows a typical calibration curve for the HE sensors. Further, as $D_{\text {mag }}$ is a function of linear actuator stepper motor position in this system, it was possible to integrate a force feedback open control loop to ensure that the actual force applied was equal to the desired force (as input by the user). At every cycle, the measured force is compared to the desired force; if the measured force is not within $\pm 0.025 \mathrm{~N}$, the shaft is incrementally adjusted (moved up or down) until the desired force is reached.

\section{User interface}

The bioreactor user interface was developed using LabView v10.0 (National Instruments Corporation). The user is prompted (Fig. 4) to input the parameters of their unique loading regimen, that is, the control mode (Force [N] or strain [\%]), frequency, duration of loading etc. and the software accordingly controls the movement of the stepper motor linear actuators to apply the required loading regimen, via an external controller and driver module.

\section{Stability, accuracy, and reproducibility}

The stability and repeatability of the integrated HE sensor system was established over a 3-week dynamic loading period, during which time, the stepper motors were cycled $(1 \mathrm{~Hz}, 30 \mathrm{~min}$ on, $1 \mathrm{~h}$ off $)$ to apply a target force of $1.2 \mathrm{~N}$. The accuracy of the sensors was determined by measuring the thickness of a selection of materials (paper [1,2, or 3 sheets] and two nylon spacers). The materials were first measured using gold standard calipers, then the same material was placed into the bioreactor chambers, and thickness was determined indirectly from magnetic field strength and the geometric algorithm developed above.

\section{Material property evaluation}

Unconfined uniaxial compression testing, using an Instron Model 5866 testing machine equipped with a 50-N load cell, was used to measure the compressive modulus of all BCseeded agarose gels, white packing foam (representative soft material), and rubber grommets (representative hard material). Samples were preloaded to $0.05 \mathrm{~N}$, allowed to equilibrate for $5 \mathrm{~min}$, and then compressed at a crosshead speed of $100 \mu \mathrm{m} / \mathrm{s}$. The tangent modulus was calculated from the linear portion of the stress-strain curves. To establish the 


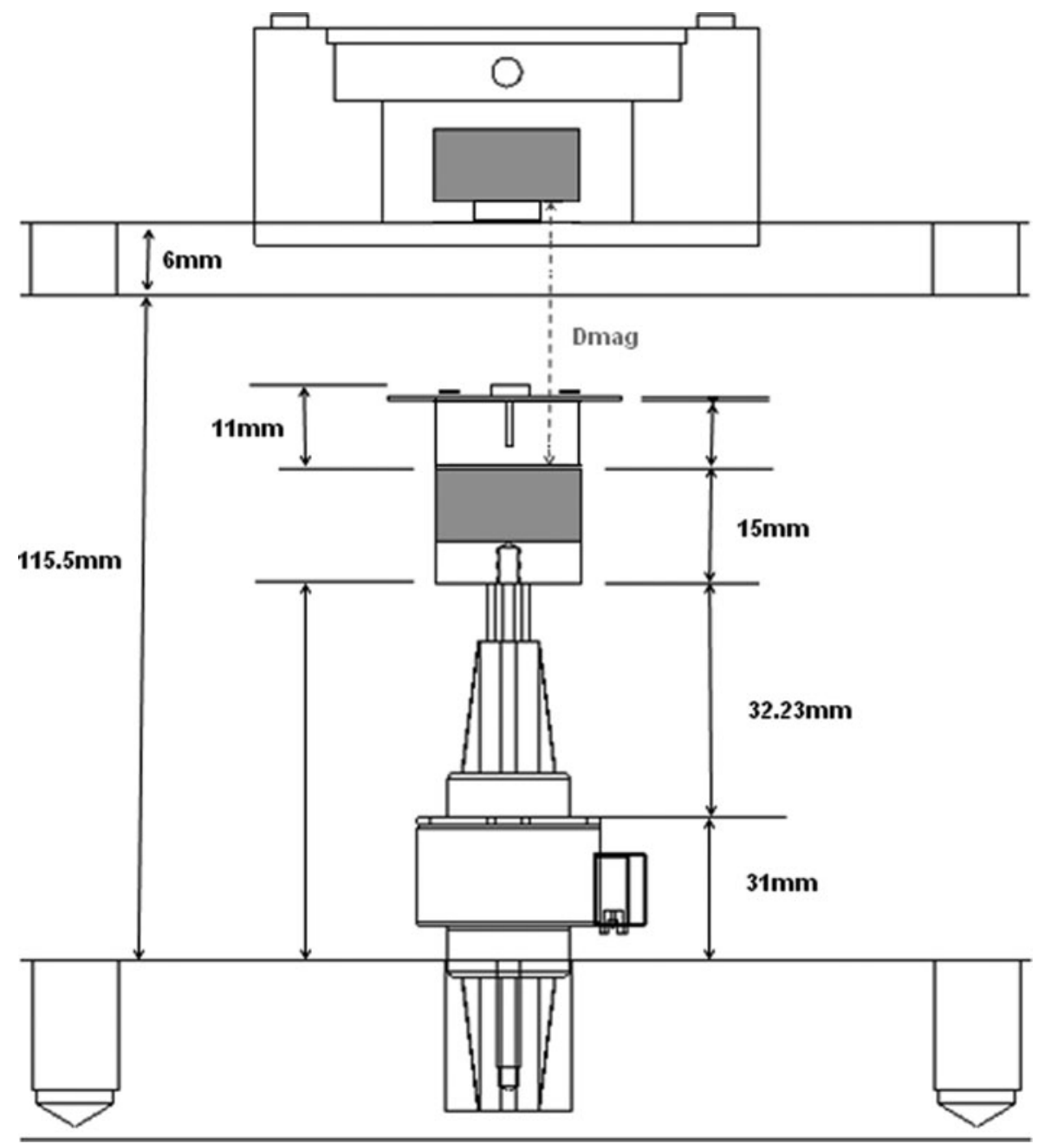

FIG. 2. Schematic depicting the geometry of the system; from the known system dimensions (shown here), it is possible to determine the distance between the magnets $\left(D_{\text {mag }}\right)$ at every position of the stepper motor (i.e., as the shaft extends and retracts). Similarly, the thickness of the construct can be determined using a simple algebraic formula, as described in the text.

compressive modulus using the bioreactor, samples were centrally positioned beneath the chamber magnet and the actuating magnet was incrementally stepped at an absolute distance of $408 \mu \mathrm{m}$ (as in the calibration program) and an average of 50 samples at each point was obtained. The re-

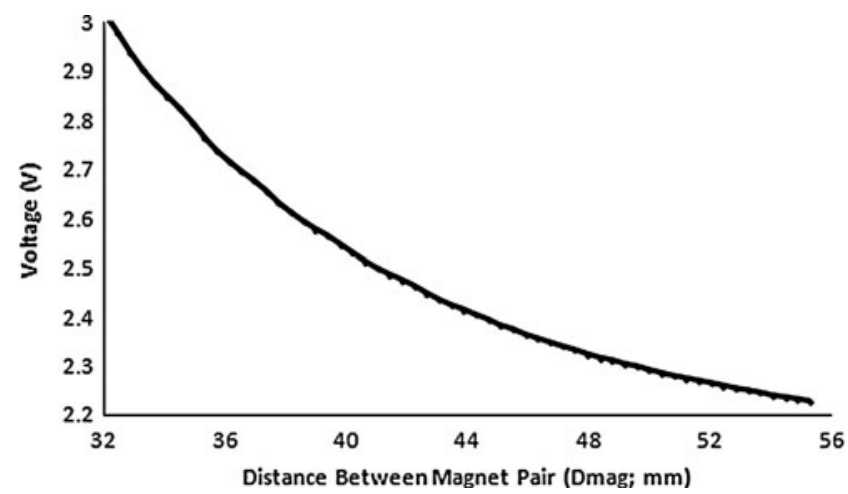

FIG. 3. Typical Hall Effect sensor calibration curve (voltage vs. distance between magnets $\left.\left[D_{\text {mag }}\right]\right)$. Sensor output voltage is proportional to the magnetic field strength, which in turn depends on the distance between magnets, $\mathrm{D}_{\text {mag }}$. Based on known geometric factors, both construct thickness and applied force can be monitored in real time as described in the text. sulting force and distance values were obtained indirectly via the algorithms previously described and the tangent modulus was calculated from the linear portion of the stressstrain curves.

\section{Statistics}

All results are expressed as mean \pm standard deviation of at least three separate experiments $(n=3)$, statistical analysis was performed using unpaired two-sided $t$-tests with a significance level of $p<0.05$.

\section{Results}

For magneto-stimulation regimens, that is, application of static/time-varying magnetic fields, the relationship between magnetic field strength and distance was determined (Fig. $5 \mathrm{~A})$; a maximum field strength of $423 \pm 41 \mathrm{mT}(n=3)$ was measured at a distance of $0 \mathrm{~mm}$, dropping to $4 \pm 1 \mathrm{mT}(n=3)$ at a distance of $50 \mathrm{~mm}$. Similarly, for mechanostimulation regimens, the force versus distance relationship between the actuating magnet pairs was measured (Fig. 5B); for a separation distance of $1 \mathrm{~mm}$, the maximum force was $117.89 \pm 5.29 \mathrm{~N}(n=5)$ decreasing to $0.25 \pm 0.01 \mathrm{~N}(n=5)$ at a separation of $50 \mathrm{~mm}$. From this graph, the physiologically relevant force range $(0.5-30 \mathrm{~N})$ was determined and the 

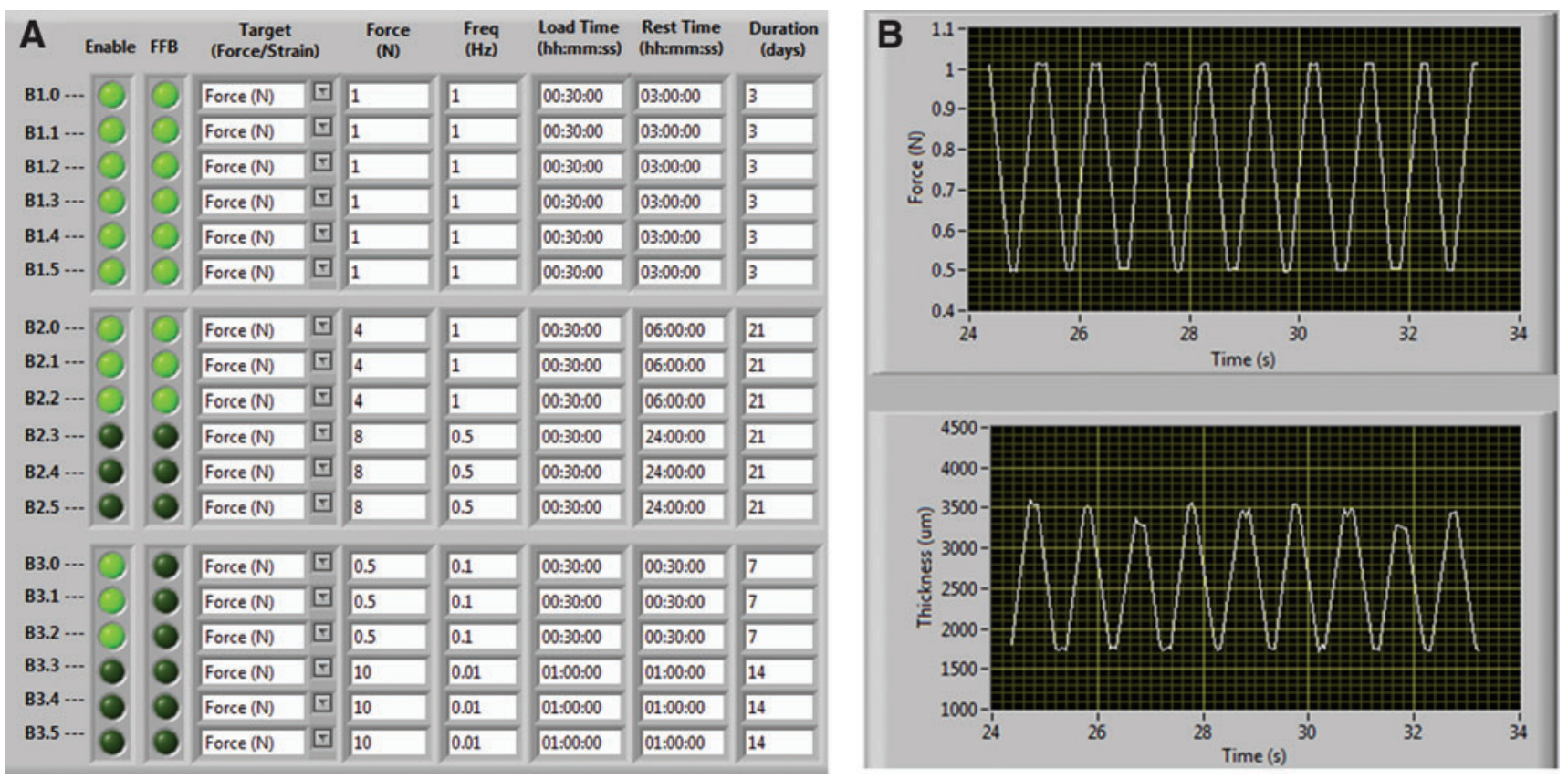

FIG. 4. The user interface for the high-throughput mageto-mechanostimulation device was developed using LabView. The control panel (A) enables users to input 18 independent magneto-mechanical loading regimens and activate force-feedback (FFB) as required. The visualization panel (B) enables the real-time data visualization of the applied force and construct thickness; this image is of one channel, and the user can select which channel they want to view at any instant. Color images available online at www.liebertpub.com/tec
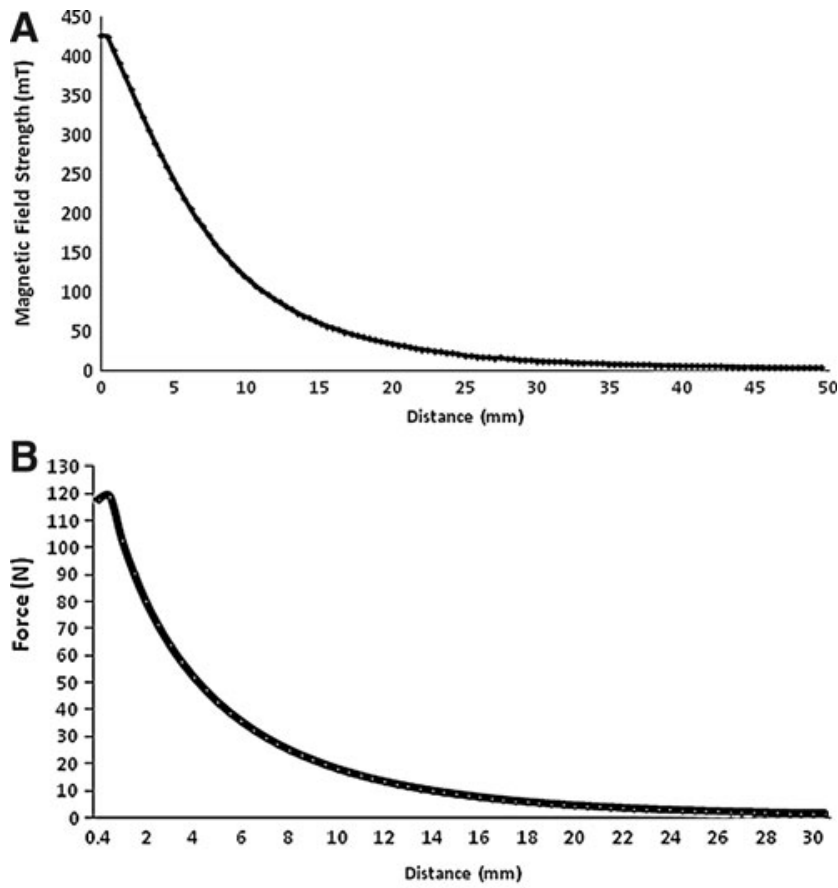

FIG. 5. For magneto-stimulation experiments, the relationship between magnetic field strength $(\mathrm{mT})$ and distance $(\mathrm{mm})$ of neodymium iron boron $(\mathrm{NdFeB})$ magnets was established (A). Similarly, for mechanostimulation experiments, the relationship between force $(\mathrm{N})$ and distance $(\mathrm{mm})$ between a pair of $\mathrm{NdFeB}$ magnets was established (B).

working distances for the geometry of the system were established.

The accuracy, repeatability, and stability of the integrated HE sensing system were evaluated via two tests. First, for accuracy assessment, rigid materials were placed in the bioreactor chambers and construct thickness, $\mathrm{T}_{\mathrm{c}}$, was measured using the HE system, after which, the same material was measured using vernier calipers to obtain a gold standard measurement. The accuracy of the HE sensors was \pm $14 \mu \mathrm{m}$ with respect to the caliper readings (Table 1). Second, the stability and repeatability of the force measurements were characterized over a 3-week dynamic loading period, with maximum variability about the $1.2 \mathrm{~N}$ set point found to be \pm 0.04 N. Further, over each half hour cycling period, the force trace was highly repeatable (Fig. 6).

The front panel of the LabView user interface is depicted in Figure 4. It can be divided into two sections: the control panel and the visualization panel. The control panel enables

Table 1. The Accuracy of the Integrated Hall

Effect (HE) Sensor System was Compared to

Measurements Made Using Gold Standard Calipers

\begin{tabular}{|c|c|c|c|}
\hline \multirow[b]{2}{*}{ Material } & \multicolumn{2}{|c|}{ Thickness ( $\mu m)$} & \multirow{2}{*}{$\begin{array}{c}\text { Average } \\
\text { difference }(\mu \mathrm{m})\end{array}$} \\
\hline & Calipers & Bioreactor & \\
\hline Paper (1 piece) & $100 \pm 0$ & $105 \pm 11$ & 5 \\
\hline Paper (1 piece) & $200 \pm 0$ & $199 \pm 8$ & -1 \\
\hline Paper (1 piece) & $300 \pm 0$ & $314 \pm 3$ & 14 \\
\hline Nylon spacer A & $1663 \pm 6$ & $1667 \pm 18$ & 4 \\
\hline Nylon spacer B & $3960 \pm 10$ & $3971 \pm 15$ & 11 \\
\hline
\end{tabular}

Values shown are the mean \pm standard deviation of $n=3$. 

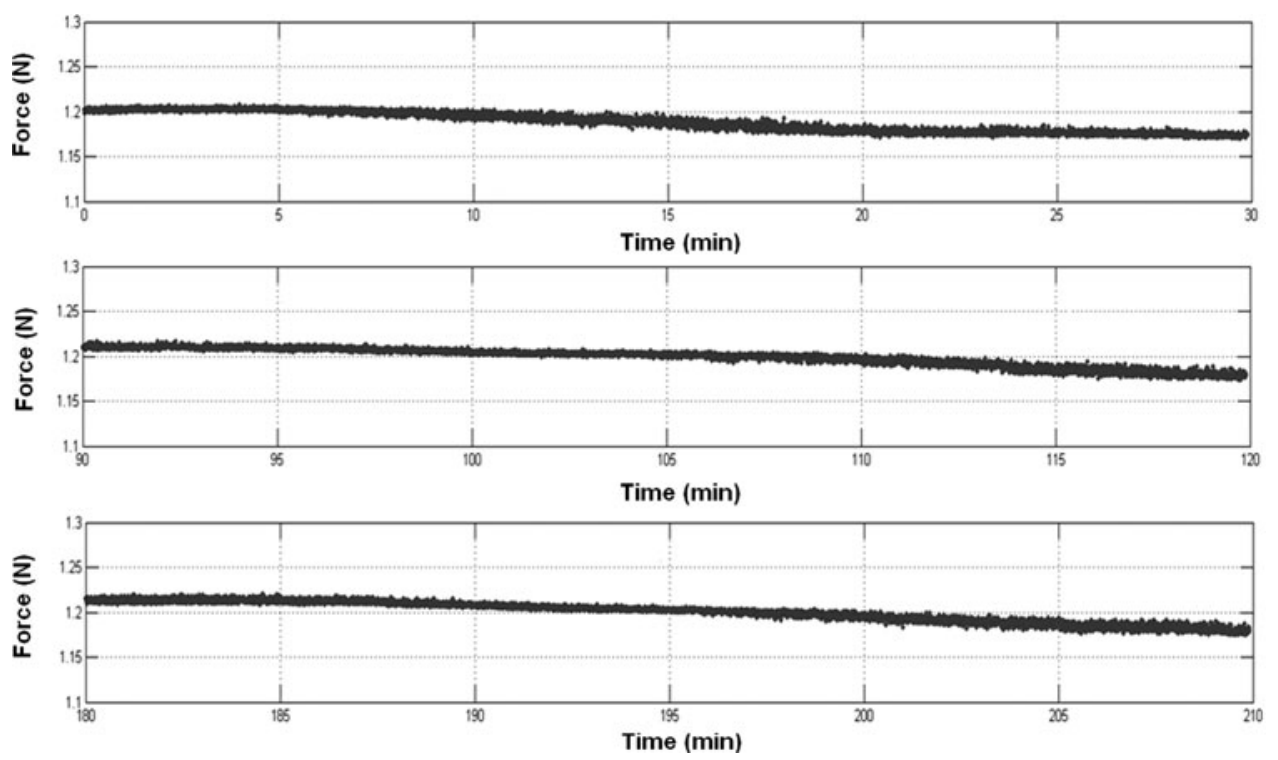

FIG. 6. Stability and repeatability was established over a 3-week dynamic loading period, during which time, the stepper motors were cycled $(1 \mathrm{~Hz}, 30 \mathrm{~min}$ on, $1 \mathrm{~h}$ off) to apply a target force of $1.2 \mathrm{~N}$. Force-time traces show three representative 30 -min cycling periods (only peak force recorded) in which, there was a maximum deviation from target force of \pm $0.04 \mathrm{~N}$. the user to input their magneto-mechanostimulation regimens (Fig. 4A). Note that multiple loading regimens can be independently implemented for each chamber, using different force amplitudes (or magnetic field strengths), stimulation frequencies, duty cycle (load time vs. rest time), and duration of loading. The visualization panel (Fig. 4B) enables the user to view the real-time force applied and construct thickness, from which, the material properties of the articular cartilage constructs can be determined as they mature in culture.

The biocompatibility of the PEI bioreactor chambers was verified by evaluating the biophysical and biochemical composition of BC-seeded agarose hydrogels. The stiffness (Fig. 7A) of BC-seeded ( 2 million/mL) agarose gels $(n=3)$ cultured in PEI chambers was not significantly $(p=0.60)$ different from those cultured in six-WP. Further, no significant difference $(p=0.82)$ was found in sGAG production (Fig. 7B; normalized to DNA) in bioreactor chambers compared to six-WP.

The final system characterization was to establish the accuracy of the bioreactor to measure material properties. Two materials were selected, a representative soft material (white packing foam) and a representative hard material (rubber grommet). The material properties were determined from the measured stress-strain curves (tangent modulus) and results (Fig. 8) showed no significant difference between the bioreactor and the Instron for both the hard rubber grommet $(705 \pm 117 \mathrm{kPa}$ and $702 \pm 20 \mathrm{kPa}$, respectively; $p=0.97)$ or the soft white packing foam $(63 \pm 11 \mathrm{kPa}$ and $65 \pm 5 \mathrm{kPa}$, respectively; $p=0.78)$.

When designing and developing new bioreactors, cost is an important factor. This device was cost effective; Table 2 reveals the cost breakdown of the individual materials that comprise the bioreactor.

\section{Discussion}

The socioeconomic burden of OA has motivated extensive research into the development of bioreactors to engineer functional articular cartilage replacement tissue. To date, despite being promising for the manufacture of implantable tissue substitutes, the goals and expectations of bioreactor development have been only partially fulfilled and most engineered tissues cultivated in bioreactors exhibit inferior biological functions and mechanical properties compared to native tissues. ${ }^{33}$ Chen and $\mathrm{Hu}^{34}$ suggested that the
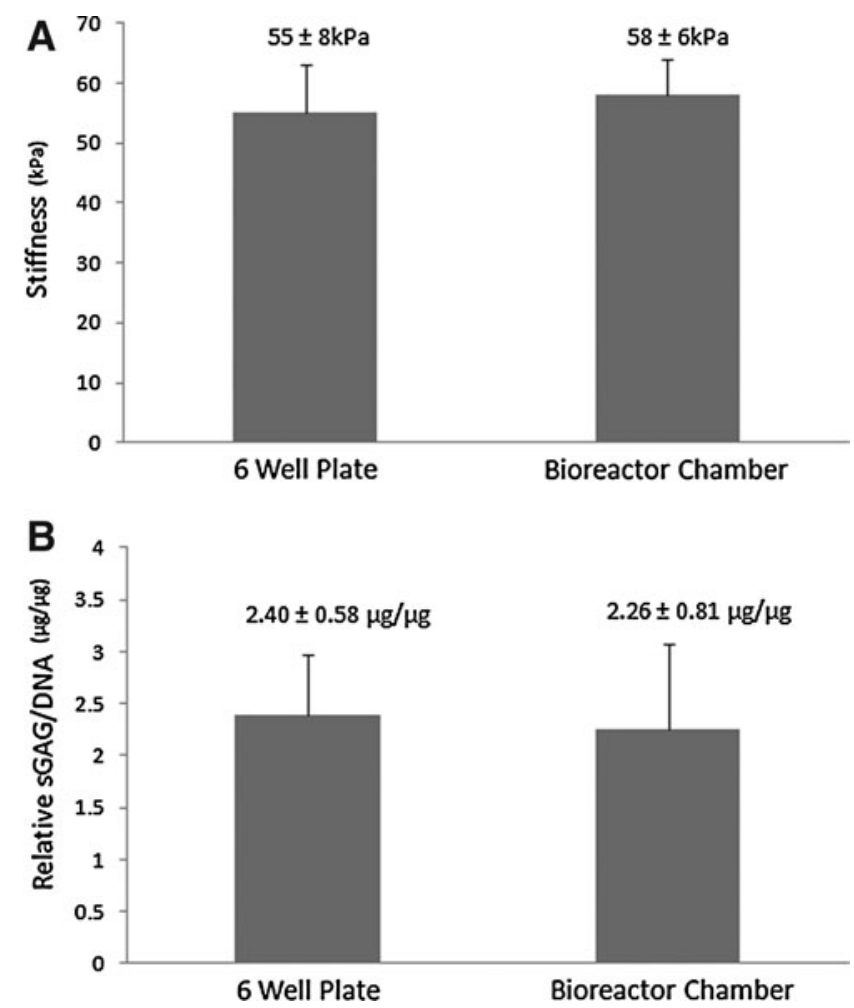

FIG. 7. Biocompatibility of the polyetherimide bioreactor chambers was assessed using standard biophysical (A; stiffness) and biochemical (B; sulfated glycosaminoglycan [sGAG] production) tests. Bovine chondrocyte (BC)-seeded $2.0 \mathrm{wt} \%$ agarose gels were cultured for 2 weeks in six-well plates and bioreactor chambers. No significant difference $(p=0.68)$ was observed in construct stiffness $(p=0.68)$ or sGAG production $(p=0.76)$. 


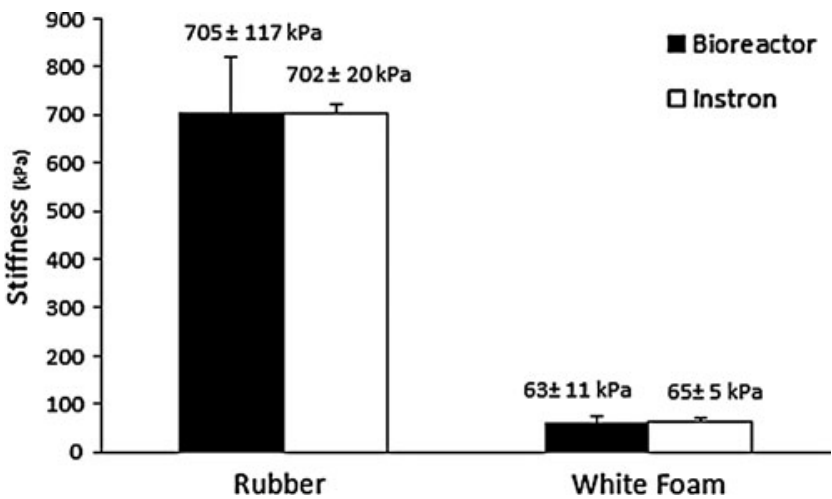

FIG. 8. Comparison of stiffness values obtained from the stress-strain curves for two representative materials; hard (Rubber) and soft (White Foam). Data were gathered by both the bioreactor and a standard materials testing (Instron) device.

development of functional tissue equivalents may require multiple mechanical cues, but currently, most bioreactors do not provide such stimuli. The high-throughput magnetomechanostimulation bioreactor developed herein was designed to provide multiple modes of biophysical stimuli, that is, magnetic and mechanical, to both tissue engineer functional replacement tissue and investigate the response of chondrocytes to multiple biophysical stimuli.

A bioreactor must create a well-defined environment in which culture conditions are regulated, including physiologically relevant environmental factors (e.g., $\mathrm{pH}, \mathrm{CO}_{2}$, and temperature), sterility and aseptic operation (e.g., sampling and feeding), and biophysical stimulation and control of the biochemical environment (e.g., media supplementation, nutrient supply, and oxygen tension). ${ }^{35}$ Bioreactor technologies should allow up-scaling, and ensure reliable and reproducible outcomes with low contamination risk and high product safety. ${ }^{1}$ Further, bioreactors should be equipped with an accurate sensing system to monitor, control, and regulate

Table 2. Bioreactor Cost List Detailing Individual Material Component Costs

\begin{tabular}{lrrr}
\hline Component & Quantity & Cost/Item & Total \\
\hline Aluminium base plates & 2 & $£ 89.40$ & $£ 178.80$ \\
Aluminium top plates & 18 & $£ 3.85$ & $£ 69.30$ \\
Gold-coated magnets & 18 & $£ 7.91$ & $£ 142.38$ \\
Teflon-coated magnets & 18 & $£ 2.12$ & $£ 38.16$ \\
Individual chambers (PEI 1000) & 18 & $£ 24.05$ & $£ 432.90$ \\
Centering devices (PEI 1000) & 18 & $£ 8.34$ & $£ 150.12$ \\
10-mm brass studding & 6 & $£ 21.16$ & $£ 126.96$ \\
Allegro HE Sensors & 40 & $£ 2.09$ & $£ 83.60$ \\
Allegro HE Sensors & 25 & $£ 2.09$ & $£ 52.25$ \\
Polypropylene press-in-plugs & 100 & $£ 0.27$ & $£ 27.23$ \\
Haydon-Kerk Linear Actuator & 18 & $£ 74.89$ & $£ 1,348.02$ \\
Trinamic motion controller & 3 & $£ 425.00$ & $£ 1,275.00$ \\
Printed circuit boards & 20 & $£ 4.40$ & $£ 88.00$ \\
& & & $£ 4,012.72$ \\
\end{tabular}

The overall bioreactor cost makes it viable for relatively widespread use.

PEI, polyetherimide. operational mechanical, biochemical, and biophysical environmental conditions. ${ }^{35}$ As the aim of tissue engineering is to improve tissue properties with cultivation time, ${ }^{16,35,36}$ the employment of techniques that can test the mechanical integrity of constructs noninvasively ${ }^{5}$ and in real time is an important design specification. All of these features were implemented in the high-throughput magneto-mechanostimulation bioreactor presented herein.

Contactless magnetic actuation, which enables completely closed and sterile culture conditions, was employed to apply static and time-varying magnetic fields and mechanical forces. Compared to many existing bioreactors that use complex mechanical (e.g., pneumatic) actuation, our noncontact actuation results in a simpler way to apply load to the construct, and is inherently easier for maintenance of sterility. To our knowledge, only three indirect compression systems have been developed, which utilize noncontact magnetically driven pressure actuators. ${ }^{10,35,36}$ However, these devices do not utilize the magnetic field as a mode of biophysical stimulation; instead, the magnetic fields are solely to actuate mechanical loading. The range of magneto-stimulation that can be applied in our system is modular and scalable, since the size and strength of the magnets can be varied without major revisions of the peripheral equipment or technology. This flexibility enables researchers to investigate a broad range of magnetic stimuli.

Electrokinetic phenomena in cartilage have previously been investigated through the application of magnetic fields. ${ }^{37-43}$ Ciombor and Aaron have made important contributions, investigating the effect of low-frequency electromagnetic fields on chondrocyte differentiation; results showed an increase in active TGF- $\beta 1,{ }^{38}$ increased expression of the matrix proteoglycans, and increased gene expression of proteoglycan and collagen II. $^{40}$ In an animal model, they showed that electromagnetic field (EMF) treatment retards the development of osteoarthritic lesions. ${ }^{41}$ We have recently shown for the first time that a static magnetic field stimulates chondrogenic differentiation of primary human bone mesenchymal stromal cells and articular cartilage cells. ${ }^{42}$ Further, we have established a dose effect, an increase in intracellular calcium, TGF- $\beta 3$ and sGAG production in response to magneto-stimulation. ${ }^{43}$ There is convincing evidence to suggest that application of an external magnetic field elicits electrokinetic events, which induce chondrogenic differentiation and maturation. However, how cells sense and respond to this biophysical stimulus, that is, the signaling pathways and mechanisms, remain to be elucidated.

The effects of mechanical stimulation on the biosynthesis of sGAG in tissue-engineered cartilage have also been extensively investigated; see for example, Darling and Athanasiou $^{47}$ for a comprehensive review. Collectively, results show an increase in sGAG production ranging from $30 \%-$ $200 \%$ (relative to unloaded controls) depending on scaffold, cell seeding density, and loading regimen. Studies investigating mechanical forces to date suggest that there are multiple regulatory pathways by which chondrocytes in articular cartilage sense and respond to mechanical stimuli, including upstream signaling pathways and mechanisms that may lead to direct changes at the level of transcription, translation, post-translational modifications, and cell-mediated extracellular assembly and degradation of the tissue matrix. $^{44}$ 
Clearly, both modes of biophysical signals (electrokinetic and mechanical) are potent promoters of chondrogenic differentiation and cartilage biosynthesis. Integration of both stimuli into one system, as in the developed highthroughput magneto-mechanostimulation bioreactor, will enable key scientific questions to be investigated, including what are the optimal parameters to engineer functional articular cartilage replacement tissues? How do chondrocytes sense and respond to biophysical stimuli? Are there distinct molecular pathways for electrokinetic and mechanical stimuli? Does this result in a synergistic production of cartilaginous ECM?

High-throughput approaches to studying biological systems have significantly increased the ability to better understand and manipulate cells for tissue-engineering and fundamental cell biology studies. ${ }^{28}$ With this in mind, our high-throughput bioreactor was built on an array-based platform designed to simultaneously and independently apply a range of physiologically relevant loading regimes. Numerous short- and long-term studies have shown that dynamic compression protocols, at moderate levels $(2 \%-15 \%$ strain; $0.5-1 \mathrm{MPa}$ ) spanning a wide range of physiological frequencies $(0.01-1.0 \mathrm{~Hz})$ can modulate chondrocyte viability, gene expression, and biosynthesis of various extracellular matrix molecules. ${ }^{44-46}$ Therefore, the force range of our device is 0.5 to $30 \mathrm{~N}$ and the frequency range is 0.01 to $1 \mathrm{~Hz}$; both the force range and loading profiles are easily adjustable and definable by the user.

The 18 independent stations have their own integrated sensing system, which enables real-time monitoring of applied force and construct thickness, as well as measurement of material properties as the articular cartilage constructs mature in culture. This offers significant benefits over other mechanical testing devices used in tissue-engineering studies, where mechanical properties are generally assessed at the end of the experiment using standard materials testing devices. ${ }^{35}$ The results of our materials testing using both the bioreactor and the Instron showed remarkable consistency, with no significant difference in the measured tangent modulus for either the soft or hard materials evaluated. Most bioreactors capable of material evaluation control specimen displacement with a mechanoelectric actuator and measure force with a load cell, expanding this standard design to multiple chambers becomes cost-prohibitive and complex for tissue-engineering applications. ${ }^{10}$ Our design is modular, scalable, and enables the determination of the biophysical properties of multiple constructs in parallel. Further, it is eminently cost effective; the integrated sensing system for one station costs $<£ 10$.

The high-throughput magneto-mechanostimulation device developed was primarily designed to apply multiple biophysical cues to multiple specimens. However, we have identified some limitations to this system that could be further developed. First, the mode of mechanical stimulation is limited to compression at the moment; we envisage a number of chamber adaptations and the use of various sized magnets to apply different modes of force, including shear, tension, and 3-point bending. Second, in its present form, this design requires the user to manually change the media during long-term culture periods; an integrated perfusion system would enable fully automated and sterile culture conditions.
In summary, the developed high-throughput magnetomechanostimulation bioreactor provides a unique platform enabling investigators to recapitulate (to some extent) the in vivo environment of the chondrocyte and investigate the combinatorial effect of electrokinetic phenomena and mechanical stimulation. Physiological understanding of dose and biological responses may be the key to new, novel treatment paradigms for $\mathrm{OA}$ and tissue-engineering strategies.

\section{Acknowledgments}

The authors acknowledge the Medical Engineering Solutions in the Osteoarthritis Centre of Excellence funded by the Wellcome Trust and the EPSRC (088844/Z/09/Z). CRE is a recipient of the Royal Society Wolfson Research Merit Award.

\section{Disclosure Statement}

No competing financial interests exist.

\section{References}

1. Schulz, R.M., and Bader, A. Cartilage tissue engineering and bioreactor systems for the cultivation and stimulation of chondrocytes. Eur Biophys J 36, 539, 2007.

2. Bitton, R. The economic burden of osteoarthritis. Am J Manag Care 15, S230, 2009.

3. Helmick, C., Felson, D., Lawrence, R., Gabriel, S., Hirsch, R., Kwoh, C.K., Liang, M.H., Kremers, H.M., Mayers, M.D., Merkel, P.A., Pillemer, S.R., Reveille, J.D., and Stone, J.H. Estimates of the prevalence of arthritis and other rheumatic conditions in the United States. Arthritis Rheum 58, 15, 2008.

4. Murphy, L., and Helmick, C.G. The impact of osteoarthritis in the United States: a population-health perspective. Am J Nurs 112, S13, 2012.

5. Korossis, S.A., Bolland, F., Kearney, J.N., Fisher, J., and Ingham, E. Bioreactors in tissue engineering. In: Ashammakhi, N., and Reis, R.L., eds. Topics in Tissue Engineering, Volume 2, Chapter 8. 2005, pp. 1-23. www.oulu.fi/spareparts/ ebook_topics_in_t_e_vol2/abstracts/korossis_0102.pdf

6. Frank, E.H., and Grodzinsky, A.J. Cartilage electromechanics-I. Electrokinetic transduction and the effects of electrolyte $\mathrm{pH}$ and ionic strength. J Biomech 20, 615, 1987.

7. Aaron, R.K., Ciombor, D.M., Wang, S., and Simon, B. Clinical biophysics: the promotion of skeletal repair by physical forces. Ann NY Acad Sci 1068, 513, 2006.

8. Li, L.P., and Herzog, W. Electromechanical response of articular cartilage in indentation-considerations on the determination of cartilage properties during arthroscopy. Computer Method Biomech 8, 83, 2005.

9. Lai, M.W., Mow, V.C., Sun, D.D., and Ateshian, G.A. On the electric potentials inside a charged soft hydrated biological tissue: streaming potential versus diffusion potential. Trans ASME 122, 336, 2000.

10. Lujan, T.J., Wirtz, K.M., Bahney, C.S., Madey, S.M., Johnstone, B., and Bottalng, M. A novel bioreactor for the dynamic stimulation and mechanical evaluation of multiple tissue-engineered constructs. Tissue Eng Part C Methods 17, 367, 2011.

11. Buschmann, M.D., Gluzband, Y.A., Grodzinsky, A.J., and Hunziker, E.B. Mechanical compression modulates matrix biosynthesis in chondrocyte/agarose culture. J Cell Sci 108, 1497, 1995. 
12. Cassino, T.R., Anderson, R., Love, B.J., Huckle, W.R., Seamans, D.K., and Forsten-Williams, K. Design and application of an oscillatory compression device for cell constructs. Biotechnol Bioeng 98, 211, 2007.

13. Davisson, T., Kunig, S., Chen, A.C., Sah, R.L., and Ratcliffe, A. Static and dynamic compression modulate matrix metabolism in tissue engineered cartilage. J Orthop Res 20, 842, 2002.

14. Demarteau, O., Jakob, M., Schafer, D., Heberer, M., and Martin, I. Development and validation of a bioreactor for physical stimulation of engineered cartilage. Biorheology 40, 331, 2003.

15. Lee, D.A., and Bader, D.L. Compressive strains at physiological frequencies influence the metabolism of chondrocytes seeded in agarose. J Orthop Res 15, 181, 1997.

16. Mauck, R.L., Soltz, M.A., Wang, C.C., Wong, D.D., Chao, P.H., Valhmu, W.B., Hung, C.T., and Ateshian, G.A. 2000. Functional tissue engineering of articular cartilage through dynamic loading of chondrocyte-seeded agarose gels. J Biomech Eng 122, 252, 2000.

17. Torzilli, P.A., Grigiene, R., Huang, C., Friedman, S.M., Doty, S.B., Boskey, A.L., and Lust, G. Characterization of cartilage metabolic response to static and dynamic stress using a mechanical explant test system. J Biomech 30, 1, 1997.

18. De Witt, M.T., Handley, C.J., Oakes, B.W., and Lowther, D.A. In vitro response of chondrocytes to mechanical loading. The effect of short term mechanical tension. Connect Tissue Res 12, 97, 1984.

19. Fukuda, K., Asada, S., Kumano, F., Saitoh, M., Otani, K., and Tanaka, S. Cyclic tensile stretch on bovine articular chondrocytes inhibits protein kinase C activity. J Lab Clin Med 130, 209, 1997.

20. Millward-Sadler, S.J., Wright, M.O., Davies, L.W., Nuki, G., and Salter, D.M. Mechanotransduction via integrins and interleukin-4 results in altered aggrecan and matrix metalloproteinase 3 gene expression in normal, but not osteoarthritic, human articular chondrocytes. Arthritis Rheum 43, 2091, 2000.

21. Wright, M.O., Nishida, K., Bavington, C., Godolphin, J.L., Dunne, E., Walmsley, S., Jobanputra, P., Nuki, G., and Salter, D.M. Hyperpolarisation of cultured human chondrocytes following cyclical pressure-induced strain: evidence of a role for alpha 5 beta 1 integrin as a chondrocyte mechanoreceptor. J Orthop Res 15, 742, 1997.

22. Frank, E.H., Jin, M., Loening, A.M., Levenston, M.E., and Grodzinsky, A.J. A versatile shear and compression apparatus for mechanical stimulation of tissue culture explants. J Biomech 33, 1523, 2000.

23. Jin, M., Frank, E.H., Quinn, T.M., Hunziker, E.B., and Grodzinsky, A.J. Tissue shear deformation stimulates proteoglycan and protein biosynthesis in bovine cartilage explants. Arch Biochem Biophys 395, 41, 2001.

24. Waldman, S.D., Spiteri, C.G., Grynpas, M.D., Pilliar, R.M., and Kandel, R.A. Long-term intermittent shear deformation improves the quality of cartilaginous tissue formed in vitro. J Orthop Res 21, 590, 2003.

25. Carver, S.E., and Heath, C.A. Increasing extracellular matrix production in regenerating cartilage with intermittent physiological pressure. Biotechnol Bioeng 62, 166, 1999.

26. Ikenoue, T., Michael, C.D., Trindade, M.C., Lee, M.S., Lin, E.Y., Schurman, D.J., Goodman, S.B., and Smith, R.L. Mechanoregulation of human articular chondrocyte aggrecan and type II collagen expression by intermittent hydrostatic pressure. J Orthop Res 21, 110, 2003.
27. Parkkinen, J.J., Ikonen, J., Lammi, M.J., Laakkonen, J., Tammi, M., and Helminen, H.J. Effects of cyclic hydrostatic pressure on proteoglycan synthesis in cultured chondrocytes and articular cartilage explants. Arch Biochem Biophys 300, 458, 1993.

28. Moraes, C., Chen, J.-H., Sun, Y., and Simmons, C.A. Microfabricated arrays for high-throughput screening of cellular response to cyclic substrate deformation. Lab Chip 10, 227, 2010.

29. Brady, M.A., Amin, H.D., Overby, D.R., and Ethier, C.R. A high-throughput mechanostimulation bioreactor for tissue engineering of articular cartilage. Abstract presented at the Biomedical Engineering Society Annual Meeting, Atlanta, GA, 2012. Abstract no. 298.

30. Massey, L.K. The Effects of Sterilization Methods on Plastics and Elastomers: The Definitive User's Guide and Databook, 2nd Ed. New York: William Andrew, Inc., 2005.

31. McCullen, S.D., Autefage, H., Callanan, A., Gentleman, E., and Stephens, M.M. Anisotropic fibrous scaffolds for articular cartilage regeneration. Tissue Eng Part A 18, 2073, 2012.

32. Popovic, R.S., Randjelovic, Z., and Manic, D. Integrated hall effect magnetic sensors. Sensors Actuators A91, 46, 2001.

33. Darling, E.M., and Athanasiou, K.M. Biomechanical strategies for articular cartilage regeneration. Ann Biomed Eng 31, 1114, 2003.

34. Chen, H.C., and Hu, Y.C. Bioreactors for tissue engineering. Biotechnol Lett 28, 1415, 2006.

35. Schulz, R.M., Wustneck, N., von donkelaar, C.C., Shelton, J.C., and Bader, A. Development and validation of a novel bioreactor system for load- and perfusion-controlled tissue engineering of chondrocyte-constructs. Biotech Bioeng 101, 714, 2008.

36. Kelly, T.A., Ng, K.W., Wang, C.C., Ateshian, G.A., and Hung, C.T. Spatial and temporal development of chondrocyte-seeded agarose constructs in free-swelling and dynamically loaded cultures. J Biomech 39, 1489, 2006.

37. Grodzinsky, A.J., Buschmann, M.D., and Gluzband, Y.A. Interaction of electromagnetic fields with chondrocyted in gel culture. Massachusetts Institute of Technology, Cambridge. Department of Electrical Engineering and Computer Science. Final Report, 14 January 1991-14 January 1992.

38. Ciombor, D.M., and Aaron, R.K. EMF stimulates cartilage differentiation in endochondral ossification conincident with and increase in TGF $\beta$ expression. In: Bersani, F., ed. Electricity and Magnetism in Biology and Medicine. New York: Kluwer/Plenum, 1999, pp. 139-144.

39. Aaron, R.K., Wang, S., and Ciombor, D.M. Upregulation of basal TGFP, levels by EMF coincident with chondrogenesis-implications for skeletal repair and tissue engineering. J Orthop Res 20, 233, 2002.

40. Ciombor, D.M., Lester, G., Aaron, R.K., Neame, P., and Caterson, B. Low frequency EMF regulates chondrocyte differentiation and expression of matrix proteins. J Orthop Res 20, 40, 2002.

41. Ciombor, D.M., Aaron, R.K., Wang, S., and Simon, B. Modification of osteoarthritis by pulsed electromagnetic field - a morphological study. Osteoarthritis Cartilage 11, 455, 2003.

42. Amin, H.D., Brady, M.A., Jing, T., McCullen, S., Stevens, M.M., Overby, D.R., and Ethier, C.R. Stimulation of chondrogenic differentiation of adult human bone marrowderived mesenchymal stromal cells (BMSC) and articular cartilage cells (ACC) using a static magnetic field (SMF) 
in vitro. Abstract presented at the Biomedical Engineering Society Annual Meeting, Atlanta, GA, 2012. Abstract no. 785.

43. Amin, H.D., Brady, M.A., St-Pierre, J.P., Stevens, M.M., Overby, D.R., and Ethier, C.R. Stimulation of chondrogenic differentiation of adult human bone marrow-derived mesenchymal stromal cells by moderate strength static magnetic fields. Tissue Engineering; Part A, 2013. DOI: 10.1089/ten.tea.2013.0307.

44. Grodzinsky, A.J., Levenston, M.E., Jin, M., and Frank, E.H. Cartilage tissue remodeling in response to mechanical forces. Annu Rev Biomed Eng 2, 691, 2000.

45. Lee, C., Grad, S., Wimmer, M., and Alini, M. The influence of mechanical stimuli on articular cartilage tissue engineering. In: Ashammakhi, N., and Reis, R.L., eds. Topics in Tissue Engineering, Volume 2, 2005, pp. 1-32. www.oulu.fi/spareparts/ ebook_topics_in_t_e_vol2/abstracts/alini_0102.pdf

46. Preiss-Bloom, O., Mizrahi, J., Elisseeff, J., and Seliktar, D. Real-time monitoring of force response measured in me- chanically stimulated tissue-engineered cartilage. Artif Organs 33, 318, 2009.

47. Darling, E.M., and Athanasiou, K.A. Articular cartilage bioreactors and bioprocesses. Tissue Eng 9, 565, 2003.

Address correspondence to: Mariea A. Brady, PhD Department of Bioengineering Imperial College London South Kensington Campus SW7 2AZ

United Kingdom

E-mail: mariea.brady@imperial.ac.uk

Received: April 9, 2013

Accepted: May 22, 2013

Online Publication Date: July 10, 2013

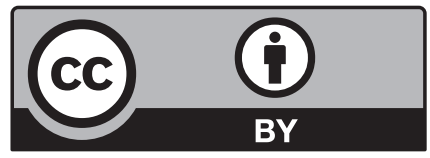

This work is licensed under a Creative Commons Attribution 3.0 United States License. You are free to copy, distribute, transmit and adapt this work, but you must attribute this work as "Tissue Engineering, Part C. Copyright 2014 Mary Ann Liebert, Inc. http://liebertpub.com/tec, used under a Creative Commons Attribution License: http://creativecommons.org/licenses/by/3.0/us/" 